\section{Hepatocellular carcinoma surveillance: Eastern and Western perspectives}

\author{
Yvonne Purcell ${ }^{1}$, Pauline Copin ${ }^{1,2}$, Luisa Paulatto ${ }^{1,2}$, Romain Pommier ${ }^{1,2}$, \\ Valérie Vilgrain ${ }^{1,2,3}$, Maxime Ronot ${ }^{1,2,3}$ \\ ${ }^{1}$ Department of Radiology, University Hospitals Paris Nord Val de Seine, Clichy; ${ }^{2}$ University \\ Paris Diderot, Sorbonne Paris Cité, Paris; ${ }^{3}$ INSERM U1149, Centre de Recherche Biomédicale \\ Bichat-Beaujon, CRB3, Paris, France
}

Eastern and Western guidelines for the management of hepatocellular carcinoma (HCC) are known to significantly differ on many points, because they reflect different diagnostic and therapeutic approaches to this cancer. Importantly, these guidelines are primarily consensusdriven when it comes to surveillance, both in term of the tests used and surveillance program design. The main difference between East and West lies in clinical practice, as several Eastern countries implement coordinated and systematic surveillance programs, while most Western countries rely on individual adherence to surveillance recommendations. This review article presents an overview of the evidence supporting surveillance programs for HCC, with a particular focus on the efficacy, cost-effectiveness, and consequences of this approach for patient survival. Western and Eastern guideline recommendations are discussed.

Keywords: Ultrasound; Surveillance; Cirrhosis; Imaging

\section{Introduction}

Hepatocellular carcinoma ( $\mathrm{HCC}$ ) is the most frequent primary liver cancer and the second most common cause of cancer-related deaths worldwide [1-3]. Like other cancers, outcomes and longterm survival are better in patients diagnosed with early-stage HCC. For example, in patients with preserved hepatic function, no evidence of portal hypertension, and single asymptomatic tumors $<5$ $\mathrm{cm}$ in diameter, surgical resection provides 5-year survival rates of 70\% [4]. Similarly, the survival rate following liver transplantation for tumors that meet the Milan criteria (solitary nodule $<5 \mathrm{~cm}$ or 3 nodules that are each $<3 \mathrm{~cm}$ in diameter) is nearly $75 \%[4,5]$. Patients with a larger tumor burden are frequently not candidates for curative treatment, and therefore their expected survival rate is lower. Unfortunately, fewer than $30 \%$ of patients are diagnosed early enough to meet criteria for tumor ablation, resection, or transplantation [6]. This supports the rationale for developing an effective surveillance program designed to improve patient management.

Surveillance programs involve closely monitoring patients with certain regularly scheduled examinations or tests. At first glance, HCC would seem to fulfill all criteria established by the World Health Organization for a surveillance program: the disease burden is an important health problem worldwide, there is a clearly identified target population (patients with cirrhosis and chronic liver

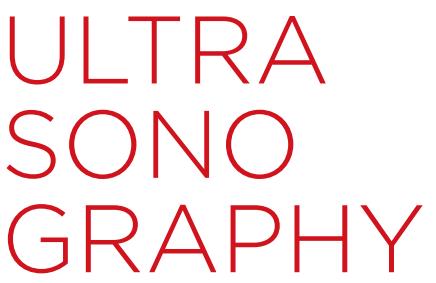

\section{REVIEW ARTICLE}

https://doi.org/10.14366/usg. 18043 pISSN: 2288-5919 • eISSN: 2288-5943 Ultrasonography 2019;38:191-199

Received: August 2, 2018 Revised: October 27, 2018 Accepted: October 28, 2018

Correspondence to: Maxime Ronot, MD, PhD, Department of Radiology, Beaujon Hospital, AP-HP, 100 Boulevard du Généaral Leclerc, 92118 Clichy, France

Tel. +33-1-40-87-55-66 Fax. +33-1-40-87-44-28 E-mail: maxime.ronot@aphp.fr
This is an Open Access article distributed under the terms of the Creative Commons Attribution NonCommercial License (http://creativecommons.org/ licenses/by-nc/4.0/) which permits unrestricted noncommercial use, distribution, and reproduction in any medium, provided the original work is properly cited.

Copyright (C) 2019 Korean Society of Ultrasound in Medicine (KSUM)

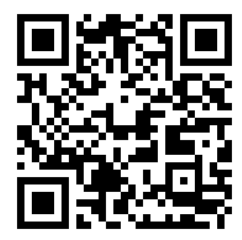

How to cite this article:

Purcell $Y$, Copin P, Paulatto L, Pommier R, Vilgrain V, Ronot M. Hepatocellular carcinoma surveillance: Eastern and Western perspectives. Ultrasonography. 2019 Jul;38(3): 191-199. 
diseases), surveillance is accepted by both patients and health providers, surveillance accuracy is acceptable, there are standardized recall procedures, surveillance is affordable, there is an advantage of treating early-stage HCC, and finally, surveillance reduces mortality.

Interestingly, while Western and Eastern guidelines significantly differ about the diagnosis and management of HCC, liver ultrasound (US) is universally considered to be a valid first-line examination technique for tumor detection and has been adopted by all guidelines [1,7-10]. However, its efficacy as a surveillance method is mainly based on the results of a single Chinese randomized controlled trial [11]. Indeed, a thorough review of the literature shows that there is a lack of robust evidence to support its benefit in reducing mortality. The main difference between Western and Eastern countries lies in the way clinical practice has adapted to this limited level of evidence. While several Eastern countries have engaged in nationwide systematic and active use of surveillance, leading to significant improvements, most Western countries still rely on individual acceptance and participation in surveillance programs, with more questionable results.

The aim of this review article is to provide an overview of the evidence supporting these surveillance programs together with their limitations, and to discuss and compare Western and Eastern positions.

\section{Eastern and Western Guidelines: More Similarities Than Differences}

\section{High-Risk Populations}

Around $90 \%$ of HCCs develop in patients with underlying risk factors, most frequently chronic viral hepatitis (types B and C), alcohol abuse, and non-alcoholic fatty liver disease (NAFLD) [6]. The epidemiology of risk factors strongly depends upon geographical factors: while most cases of HCC are associated with hepatitis B $(60 \%)$ in several Eastern and Southeastern Asian countries, chronic hepatitis $C$ appears to be a major risk factor in Japan and in Western countries [4], while excessive alcohol consumption is most frequent in Western countries, and the contribution of NAFLD is increasing, especially in North America $[2,12]$.

\section{Patients with cirrhosis}

Most patients with $\mathrm{HCC}$ are diagnosed at the stage of cirrhosis, and approximately one-third of patients with cirrhosis will develop HCC during their lifetime [13], corresponding to a risk of approximately $1 \%-8 \%$ per year. It is important to note that this risk varies depending on the etiology of cirrhosis and the possible control of its cause. This rate is around $2 \%$ in patients with hepatitis $B$ virus (HBV)-related cirrhosis, and up to $3 \%-8 \%$ in hepatitis $C$ virus (HCV)-related cirrhosis in the absence of viral control or eradication, respectively [14]. According to the Japanese guidelines, this justifies identifying patients with cirrhosis and HBV or HCV infection as "super-high-risk" patients (Table 1) [9]. However, successful viral eradication leading to a sustained virological response in HCV and HBV patients significantly decreases, but does not eliminate, the risk of HCC $[15,16]$. Other factors, such as male sex, age, and the presence of portal hypertension have been shown to increase the risk of HCC in patients with cirrhosis. However, to date, these factors have not been incorporated into recommendations, and all patients with cirrhosis should be included in HCC surveillance programs, whatever the control or severity of the underlying cause of liver disease (level of evidence, moderate; level of recommendation, high).

\section{Non-cirrhotic patients}

Although the actual yearly incidence has not been clearly identified,

Table 1. High-risk population candidates for surveillance programs according to Western and Eastern guidelines

\begin{tabular}{|c|c|c|c|c|c|}
\hline & \multicolumn{2}{|c|}{ Western } & \multicolumn{3}{|c|}{ Eastern } \\
\hline & AASLD (8) & EASL (1) & JSH (9) & APASL (7) & $\operatorname{KLCA}(10)$ \\
\hline Super-high-risk patients & - & - & Cirrhosis with HBV or HCV & - & - \\
\hline \multirow[t]{3}{*}{ High-risk patients } & Cirrhosis $^{a)}$ & Cirrhosis $^{\mathrm{a})}$ & Cirrhosis of any cause & Cirrhosis with HBV or HCV & Cirrhosis of any cause \\
\hline & & $\mathrm{HBV}^{\mathrm{b})}$ & HBV & & $\mathrm{HBV}$ \\
\hline & & F3 & $\mathrm{HCV}$ & & $\mathrm{HCV}$ \\
\hline
\end{tabular}

AASLD, American Association for the Study of Liver Diseases; EASL, European Association for the Study of the Liver; JSH, Japanese Society of Hepatology; APASL, Asian Pacific Association for the Study of the Liver; KLCA, Korean Liver Cancer Association; HBV, hepatitis B virus; F3, fibrosis stage 3 according to the METAVIR system; HCV, hepatitis C virus; HCC, hepatocellular carcinoma.

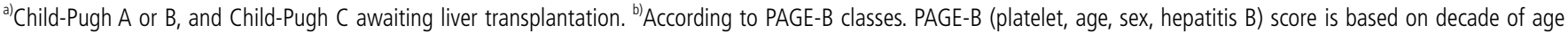
$(16-29,0 ; 30-39,2 ; 40-49,4 ; 50-59,6 ; 60-69,8 ;$ and $\geq 70,10)$, sex (male 6, female 0) and platelet count $(\geq 200,000 / \mu \mathrm{L}, 0 ; 100,000-199,999 / \mu \mathrm{L}, 1 ;$ and $<100,000 / \mu \mathrm{L}, 2)$ : a total sum of $\leq 9$ is considered to indicate a low risk of HCC (almost $0 \% \mathrm{HCC}$ at 5 years), a score of $10-17$ to indicate intermediate risk ( $3 \%$ incidence of HCC at 5 years) and $\geq 18$ to indicate high risk ( $17 \%$ incidence of $\mathrm{HCC}$ at 5 years). 
patients with chronic active HBV infection are at risk of HCC even in the absence of cirrhosis. It is even recommended to screen specific subgroups of inactive HBV carriers such as African males born in Africa because of aflatoxin-B1 exposure [17]. Fewer studies have been conducted on $\mathrm{HCV}$, but the available data suggest that patients with advanced fibrosis have a high risk of HCC $[18,19]$.

Data on the incidence of HCC in non-cirrhotic patients with nonviral chronic liver diseases, such as NAFLD, autoimmune liver disease, hemochromatosis, or others, are also limited [1,12]. The risk in patients with NAFLD is probably not limited to those with advanced fibrosis or cirrhosis $[12,20]$. However, the uncertainty regarding the actual incidence of HCC in cases of NAFLD without cirrhosis, which occur at high rates in Western (and now even Eastern) populations, explains why guidelines on HCC surveillance recommend following current practices and only performing surveillance in patients with cirrhosis (level of evidence: low) [12,21]. Moreover, this population also has more competing causes of mortality than patients with viral hepatitis, which may impact the efficacy of surveillance

\section{Evidence Supporting Surveillance Programs}

\section{Benefits of surveillance programs}

The extant evidence on the efficacy of surveillance programs is mainly based on the results of a Chinese randomized controlled trial. This population-based cluster study randomized surveillance (measurement of a-fetoprotein [AFP] levels and liver US performed every 6 months) versus no surveillance in entire villages of patients with HBV and reported a 37\% reduction in HCC-related mortality in the surveillance group, despite a low rate of participation (55\%) [11]. Importantly, not all patients included in this study were cirrhotic. Therefore, the target population was different from the target population advocated in most programs. In fact, no randomized controlled trials have been conducted on cirrhotic populations.

Since this study, close to 40 observational studies have been published, showing that surveillance enables the identification of early-stage HCC and increases the number of patients receiving curative treatment. The 2018 guidelines issued by the American Association for the Study of Liver Diseases summarize this literature [8]. The authors report an odds ratio (OR) of 2.11 (95\% confidence interval [CI], 1.88 to 2.33) of surveillance for the early detection of HCC (from 10,904 patients in 38 observational studies), and an OR of $2.24(95 \% \mathrm{Cl}, 1.99$ to 2.52$)$ for curative treatment (from 24,373 patients in 35 observational studies) [8]. The authors also reported an $\mathrm{OR}$ of 1.90 (95\% Cl, 1.67 to 2.17$)$ for 3-year overall survival (from 10,850 patients in 23 studies) [8].

Yet, these studies should be interpreted with caution because they

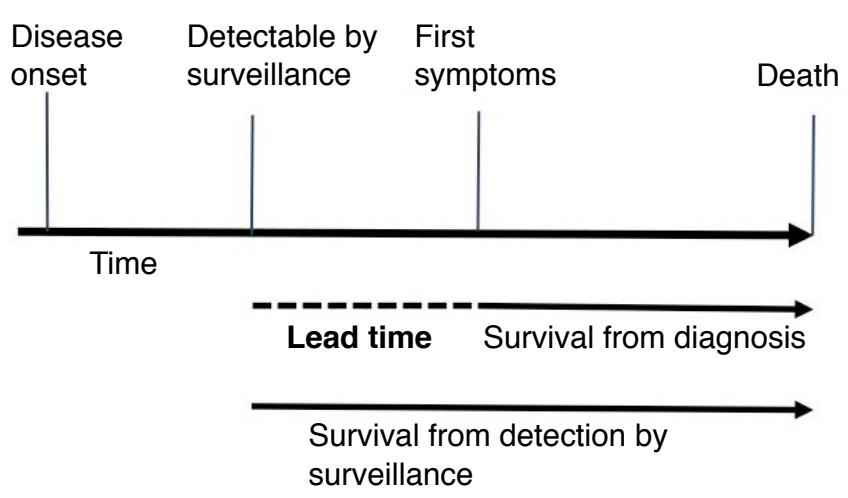

Fig. 1. Illustration of the lead-time bias. Lead time is the length of time between an early diagnosis with surveillance and the time at which the diagnosis would have been made without screening. The lead-time bias corresponds to a perceived longer survival time due to surveillance, even if surveillance does not actually affect the course of the disease.

suffer from the methodological biases of cohort studies. That is, they are not representative of cirrhotic populations as a whole, and, more importantly, they are subject to lead-time bias and length-time bias.

\section{Lead-time bias}

Lead time is the length of time between an early diagnosis with surveillance and the time at which the diagnosis would have been made without screening. The lead-time bias corresponds to a perceived longer survival time due to surveillance, even if surveillance does not actually affect the course of the disease (Fig. 1). A recent meta-analysis by Singal et al. [22], including 47 studies with 15,158 patients and $6,284(41.4 \%)$ with HCC detected by surveillance, reported improved early-stage detection (OR, 2.08; $95 \% \mathrm{Cl}, 1.80$ to 2.37$)$ and curative treatment rates $(\mathrm{OR}, 2.24 ; 95 \%$ $\mathrm{Cl}, 1.99$ to 2.52), as well as significantly prolonged survival (OR, 1.90; $95 \% \mathrm{Cl}, 1.67$ to 2.17 ), which remained significant in the subset of studies that adjusted for lead-time bias. New data are emerging based on a study of large prospective cohorts of patients with cirrhosis included in surveillance programs that support these results [23]. They show that a benefit to survival related to compliance with HCC surveillance guidelines is expected, in particular after correction for lead-time bias and assumptions regarding tumor doubling time [23]. Nevertheless, the real effect of surveillance on survival for populations undergoing US surveillance remains subject to ongoing debate. Very recently, Moon et al. [24] published a matched casecontrol study and found that screening patients with cirrhosis for HCC by US, measurements of serum AFP levels, either test, or both was not associated with decreased HCC-related mortality. 
One explanation may be that the real effect on survival is limited. Recently, Cadier et al. [25] reported a mean gain of 0.37 years. This may seem rather low, but it has to be compared to the short median overall survival of patients with HCC (e.g., 9.4 months, or 0.78 years, in the recent national French observational study by Goutte et al. [26]).

\section{Cost-effectiveness}

Cost-effectiveness analysis is a form of economic analysis that compares the relative costs and outcomes (effects) of different courses of action. For HCC surveillance, cost-effectiveness analysis should compare the gain in quality-adjusted life expectancy obtained following surveillance to the total costs of the program. However, it is important to note that the former depends upon the incidence of HCC and the possibility of providing curative management to patients diagnosed with HCC, while the latter includes the costs of surveillance tests, as well as false positive cases, tumor diagnosis, staging, and of course, treatment.

Studies show that an incidence of HCC of at least 1.5\% per year would justify surveillance in HCC in patients with cirrhosis [27], whatever the etiology. However, the surveillance of Child-Pugh class C patients, except those awaiting liver transplantation, is probably not cost-effective due to the difficulty of performing secondary curative treatment $[28,29]$. In 2012, Ruggeri [30] published a systematic review of seven studies providing a full cost-effectiveness evaluation of different HCC surveillance techniques. These results showed that US, alone or in association with AFP, was likely to be the most cost-effective strategy. Indeed, although this strategy has been consistently reported to be the most effective program for early tumor diagnosis, it is also associated with increased costs compared to longer testing intervals (i.e., 12 months or more). This extra cost is compensated by the lower cost of secondary treatments. Indeed, the cost of surveillance and diagnosis only represents $10 \%$ $20 \%$ of the total costs of cancer management $[31,32]$. This was recently confirmed in a study performed in France and the United States based on the assessment of Markov models and transition probabilities derived from academic and real-life data obtained in longitudinal cohorts [25].

Finally, and importantly, previous results may not be easily extrapolated to patients with NAFLD. Indeed, in this setting, computed tomography (CT) or magnetic resonance imaging (MRI) is more frequently performed after a first incomplete US examination of the liver. As a consequence, although surveillance based on regular US is considered cost-effective in patients at risk for HCC, that may no longer be the case if MRI or CT scans have to be performed. Whether or not the abovementioned short MRI scanning protocols may replace US in this context requires further large-scale investigation.

\section{Surveillance Modality}

\section{Tests to be used}

It is important to distinguish surveillance tests from diagnostic tests. The former aim at detecting focal lesions in the liver, whatever their final nature, while the latter aim at providing a definitive characterization of those nodules (i.e., whether a nodule is HCC). The guidelines suggest using two main types of tests for HCC surveillance: serological and imaging examinations (performed either separately or in combination). The most widely accepted imaging modality, which is present in and recommended by all guidelines, is US (level of evidence, moderate; level of recommendation, high). It is inexpensive, accessible, and well accepted by patients, and it has an acceptable diagnostic accuracy. It is also frequently the only imaging method available in developing countries or remote rural areas. The reported sensitivity of this test ranges from $58 \%$ to $89 \%$, with an excellent specificity (above 90\%) [33]. A meta-analysis including 13 prospective studies showed that US surveillance was accurate for the detection of all types of HCC before it was clinically detectable with a pooled sensitivity of $94 \%$. However, US was less effective for detecting early-stage HCC, with a sensitivity of only $63 \%$ [34]. Tzartzeva et al. [35] recently published another metaanalysis including 32 studies (13,367 patients) and reported lower sensitivities. US detected any-stage HCC with $84 \%$ sensitivity $(95 \%$ $\mathrm{Cl}, 76 \%$ to $92 \%$ ), but early-stage HCC with only $47 \%$ sensitivity (95\% Cl, 33\% to 61\%) [24]. This low sensitivity is explained by patient-related factors that may limit US exploration of the liver (obesity, marked steatosis, heterogeneous cirrhotic liver parenchyma, and poor cooperation) and tumor-related features (location, infiltrative forms, and tumor echogenicity). The fact that obesity and steatosis compromise the completeness of an US examination of the liver is clinically important in the context of the increasing epidemic of NAFLD. It shows that results derived from populations with other chronic liver diseases may not be easily extrapolated to patients with NAFLD. Technical limitations and the radiologist's skill and expertise play also an important role. Indeed, a recent Japanese study with US performed by highly skilled radiologists reported detection of tumors with a mean diameter of $1.6 \pm 0.6 \mathrm{~cm}$ [36].

The role of other imaging techniques has not been thoroughly investigated. A recent randomized study comparing twice-a-year US to once-a-year triple-phase-contrast CT reported that US was slightly more sensitive and less expensive for the detection of early HCC [37]. Furthermore, the use of CT requires ionizing radiation, which can lead to a substantial cumulative dose in populations requiring regular screening. A recent meta-analysis reported a sensitivity of 
Table 2. Surveillance modalities according to Western and Eastern guidelines

\begin{tabular}{|c|c|c|c|c|c|}
\hline \multirow{2}{*}{ Patient group } & \multicolumn{2}{|c|}{ Western } & \multicolumn{3}{|c|}{ Eastern } \\
\hline & AASLD (8) & EASL (1) & JSH (9) & APASL (7) & KLCA (10) \\
\hline \multicolumn{6}{|l|}{ Modality } \\
\hline Super-high and high-risk patients & Liver US \pm AFP & Liver US & $\begin{array}{l}\text { Liver US+AFP/AFP-L3, } \\
\text { PIVKA-II } \\
\text { CT/EOB-MRI }\end{array}$ & $\begin{array}{l}\text { Liver US } \\
\text { AFP' }^{\text {a) }}\end{array}$ & $\begin{array}{l}\text { Liver US } \\
\text { AFP }^{b)}\end{array}$ \\
\hline \multicolumn{6}{|l|}{ Interval } \\
\hline Super-high-risk patients & - & - & $\begin{array}{l}3-4 \mathrm{mo} \\
\text { CT/MRI 6-12 mo }\end{array}$ & - & - \\
\hline High-risk patients & $6 \mathrm{mo}$ & $6 \mathrm{mo}$ & $\begin{array}{l}6 \text { mo } \\
\text { No CT/EOB-MRI }\end{array}$ & $6 \mathrm{mo}$ & $6 \mathrm{mo}$ \\
\hline
\end{tabular}

AASLD, American Association for the Study of Liver Diseases; EASL, European Association for the Study of the Liver; JSH, Japanese Society of Hepatology; APASL, Asian Pacific Association for the Study of the Liver; KLCA, Korean Liver Cancer Association; US, ultrasound; AFP, a-fetoprotein; L3, ratio of glycosylated AFP to total AFP; PIVKA-II, prothrombin induced by vitamin $\mathrm{K}$ absence II; CT, computed tomography; EOB, ethoxybenzyl (gadoxetic acid); MRI, magnetic resonance imaging.

${ }^{\text {a) }}$ Accepted diagnostic cutoff value $>200 \mathrm{ng} / \mathrm{mL}$, even though the measurement is not recommended. ${ }^{\text {b) }}$ For nodules $<1 \mathrm{~cm}$.

$84 \%$ with gadoxetic acid-enhanced MRI for the detection of all HCC [38]. Nevertheless, despite its better results, MRI is not suitable for surveillance programs because a complete MRI examination takes between 30 and 45 minutes. Therefore, the use of short scanning protocols that take less than 10 minutes has been suggested; such protocols reduce the number of acquired sequences and concentrate on the most sensitive sequences for tumor detection (T2-weighted, diffusion-weighted, and T1-weighted during the hepatobiliary phase). This was supported by recent retrospective studies showing that "simulated" fast MRI protocols (by limiting interpretation to selected sequences) could be an acceptable alternative to US [3941].

The use of serological markers is more controversial (level of evidence: low). AFP has been the most widely investigated. More recently, des-gamma-carboxy prothrombin, also known as prothrombin induced by vitamin $\mathrm{K}$ absence II (PIVKA II), and the ratio of glycosylated AFP (L3 fraction) to total AFP have been analyzed $[42,43]$. The results of AFP as a test for surveillance are poor because only $10 \%-20 \%$ of cases of early HCC express AFP. Although the combination of US and AFP has been estimated to increase the sensitivity for early HCC by $6 \%$ compared to US alone, the rate of false positive results is also increased, with negative consequences on direct and indirect costs for each early HCC detected $[44,45]$. This explains why Western guidelines do not recommend systematically measuring AFP for surveillance $[1,8]$. However, the recent metaanalysis from Tzartzeva et al. [35] questions this approach. Indeed, in studies comparing US with and without AFP measurements, US alone detected any-stage HCC with a lower level of sensitivity than US with AFP measurement (relative risk [RR], $0.88 ; 95 \% \mathrm{Cl}, 0.83$ to 0.93) and early-stage HCC with a lower level of sensitivity than US with AFP measurements (RR, $0.81 ; 95 \% \mathrm{Cl}, 0.71$ to 0.93 ). However,
US alone detected HCC with a higher level of specificity than US with AFP measurements (RR, 1.08; $95 \% \mathrm{Cl}, 1.05$ to 1.09). US with AFP measurements detected early-stage HCC with $63 \%$ sensitivity (95\% Cl, 48\% to $75 \%$ ), while US without AFP measurements did so with $45 \%$ sensitivity $(95 \% \mathrm{Cl}, 30 \%$ to $62 \%)(\mathrm{P}=0.002)$.

In line with these results, Eastern guidelines take a different approach and still propose a combination of US and serum markers (Table 2) $[7,9,10]$. The most extensive program is that of the Japanese Society of Hepatology, which recommends US and the measurement of tumor markers (including AFP, AFP-L3, and PIVKAII) for both super-high-risk and high-risk populations [9]. Interestingly, these guidelines also propose periodic imaging with dynamic CT or preferably dynamic gadoxetic-enhanced MRI for super-high-risk patients and those in whom US evaluation is difficult due to a very coarse background liver parenchyma caused by cirrhosis and/or obesity.

\section{Surveillance Interval}

Based on the expected doubling time of HCC, all guidelines recommend surveillance at 6 -month intervals, except the Japanese guidelines, which also recommend a 3- to 4-month interval for super-high-risk populations (Table 2) [16]. In this setting, CT or MRI should be performed every 12 months. The recommended 6-month interval for US surveillance is based on the results of a large French randomized multicenter study that did not show any benefit to shorter surveillance intervals [46]. This study showed that a 3-month interval did not significantly increase the likelihood of detecting small $(\leq 3 \mathrm{~cm}) \mathrm{HCCs}(79 \%$ vs. $70 \%)$, or improve the amenability to curative treatment $(62 \%$ vs. $58 \%)$ or 5 -year survival $(85 \%$ vs. $86 \%)$. On the other hand, the 3 -month interval was associated with a higher risk of detecting non-malignant lesions than the 6-month 
interval, leading to an increased cost of recall procedures. The results of comparisons of patient cohorts monitored every 6 or 12 months were either similar, or a lower survival rate was reported with the 12-month interval [47-49]. Finally, the meta-analysis by Singal et al. [34] mentioned above showed that the pooled sensitivity of US-based surveillance decreases to around 50\% with an annual program.

\section{Limitations of Surveillance Programs: The Western Perspective}

\section{Limited program uptake}

In a prospective cohort study including 1,671 patients with biopsyproven viral cirrhosis who underwent surveillance for HCC, Costentin et al. [23] showed that overall survival was longer in patients compliant with surveillance guidelines than in non-compliant patients, after lead-time adjustment. However, the main limitation of surveillance programs is their limited uptake. Importantly, most data come from Western studies. Several Western studies have shown that the proportion of patients eligible for surveillance who actually participate in the program as planned was low. Davila et al. [50] reported that no more than $28 \%$ of patients diagnosed with HCC had undergone at least 1 US surveillance test in the 3 years preceding diagnosis. The same group reported a study in a larger population of at-risk patients and confirmed the low rate of surveillance, with only $12 \%$ and $59 \%$ of patients undergoing consistent and inconsistent surveillance, respectively [51]. This calls the effectiveness of surveillance programs into question.

If efficacy is a measure of the degree to which a procedure obtains the expected result under standardized conditions, effectiveness measures the extent of benefit when the procedure is applied to clinical practice [52]. Therefore, the latter includes nonstandardized factors corresponding to the "real-world" experience of both patients and health providers.

\section{Factors associated with program uptake}

Factors may include convincing and recommendations by physicians, patient acceptance and adherence, and the organization of the healthcare system, as well as economic, cultural, and social influences. It is important to stress that the most important factor is the failure of the referring physician to prescribe surveillance in high-risk patients. Several studies have tried to identify other factors. Davila et al. [51] demonstrated that being followed by a gastroenterologist/hepatologist or an academic physician was associated with a higher likelihood of receiving surveillance than being followed by a primary care physician. This was recently confirmed by Goldberg et al. [53] in a series of 26,577 patients.
Farvardin et al. [54] tried to identify the association between surveillance and patient knowledge, attitudes, and perceived barriers in a racially diverse and socioeconomically disadvantaged cohort of patients with cirrhosis. They showed that around $50 \%$ of the patients believed that eating a healthy diet made HCC surveillance unnecessary, and that close to $35 \%$ believed that HCC surveillance was not necessary if they had a normal physical exam and/or lacked clinical symptoms. Overall, nearly half the patients were found to have barriers to receiving HCC surveillance, including difficulty with the scheduling process, costs, and transportation difficulties. Goldberg et al. [53] also reported an inverse relationship between US lead time and the odds of having the test performed (OR, 0.77 ; $95 \% \mathrm{Cl}, 0.72$ to 0.82 when ordered $>180$ days ahead of time; and $\mathrm{OR}, 0.90 ; 95 \% \mathrm{Cl}, 0.85$ to 0.94 if the lead time was $91-180$ days).

\section{Harm caused by surveillance programs}

Atiq et al. [55] suggested that another explanation for the equivocal benefits of surveillance programs was the possible harm that they cause, defined as any follow-up tests (CT, MRI, liver biopsy, angiogram) performed for false-positive or indeterminate surveillance results. In a series of 680 patients with cirrhosis who underwent surveillance by US and AFP measurements, surveillancerelated physical harm was observed in over one-fourth of the patients, with a higher proportion of US-related than AFP-related harm [55]. Even though harm was mild to moderate in most patients, these data clearly show that the use of surveillance is insufficient and suboptimal in the real world of healthcare and should stimulate educational policies to expand knowledge and for the correct use of this tool. Radiologists who frequently perform liver US need to be more aware of these unmet needs and help address them. Thus, all initiatives to optimize compliance based on procedures to call patients enrolled in HCC surveillance programs (including traditional approaches such as mail outreach or newer communication strategies) are welcome, because they have been shown to increase the rate of patients who undergo their next US surveillance examination [56].

\section{Addressing the limitations: the Japanese experience}

Since large observational studies are, and will remain, limited by methodological biases, another possible way of demonstrating the effect of surveillance is to look at countries that have implemented nationwide active and systematic surveillance policies, such as Japan.

Nationwide surveillance for the early detection of HCC has become common practice in Japan for over 30 years. Since the 1990s, the Japanese Society of Hepatology has promoted public awareness of the importance of HCC surveillance by appointing 
dedicated educators in all Japanese regions. The government has also actively engaged in preventative measures against viral hepatitis and HCC (free testing for hepatitis virus, surveillance covered by the national health insurance and social insurance system). As a result, the majority of cases of HCC are diagnosed at an early stage (62\% according to Kudo [57]), and the 5-year survival rate has been reported to be as high as $43 \%$. South Korea has been operating a similar surveillance program since 2003. As a result, the survival rate has gradually improved from the early 1990s (10.7\%) to the late 2000s (26.7\%) [58]. In Western countries, even though surveillance is clearly recommended by guidelines, its efficacy has been debated more, and clinical practice still relies on individual participation and compliance with the surveillance program.

\section{Conclusion}

HCC surveillance based on regular US examination of the liver, alone or in combination with serum markers, appears to be beneficial and cost-effective. It leads to the detection of earlier-stage disease, a higher rate of curative treatment and, probably, improved survival. This strategy has been endorsed by both Western and Eastern guidelines on HCC surveillance despite a limited level of evidence. Nevertheless, daily practice significantly differs in Western and Eastern countries. In Western countries, surveillance is debated, patient uptake is lower, and participation in surveillance programs relies mostly on individual compliance, rather than nationwide structured surveillance. In Eastern countries such as Japan and South Korea, nationwide surveillance programs have been implemented, and the reported cancer-related survival is significantly higher than that of Western countries. Significant collective efforts in terms of education of physicians, patient acceptance and adherence, and organization of the healthcare system must be undertaken, with due consideration of economic, cultural, and social influences.

ORCID: Yvonne Purcell: https://orcid.org/0000-0003-1118-7053; Romain Pommier: https://orcid.org/0000-0002-9481-0017; Valérie Vilgrain: https://orcid.org/00000002-3568-7725; Maxime Ronot: https://orcid.org/0000-0001-7464-3939

\section{Conflict of Interest}

No potential conflict of interest relevant to this article was reported.

\section{References}

1. European Association for the Study of the Liver. EASL Clinical Practice Guidelines: management of hepatocellular carcinoma. J Hepatol 2018;69:182-236.
2. El-Serag HB. Hepatocellular carcinoma: recent trends in the United States. Gastroenterology 2004;127(5 Suppl 1):S27-S34.

3. El-Serag HB, Rudolph KL. Hepatocellular carcinoma: epidemiology and molecular carcinogenesis. Gastroenterology 2007;132:25572576.

4. Llovet JM, Bruix J. Early diagnosis and treatment of hepatocellular carcinoma. Baillieres Best Pract Res Clin Gastroenterol 2000;14: 991-1008.

5. Mazzaferro V, Regalia E, Doci R, Andreola S, Pulvirenti A, Bozzetti F, et al. Liver transplantation for the treatment of small hepatocellular carcinomas in patients with cirrhosis. N Engl J Med 1996;334:693699.

6. Marrero JA, Fontana RJ, Barrat A, Askari F, Conjeevaram HS, Su $\mathrm{GL}$, et al. Prognosis of hepatocellular carcinoma: comparison of 7 staging systems in an American cohort. Hepatology 2005;41:707716.

7. Omata M, Lesmana LA, Tateishi R, Chen PJ, Lin SM, Yoshida H, et al. Asian Pacific Association for the Study of the Liver consensus recommendations on hepatocellular carcinoma. Hepatol Int 2010;4:439-474.

8. Heimbach JK, Kulik LM, Finn RS, Sirlin CB, Abecassis MM, Roberts $L R$, et al. AASLD guidelines for the treatment of hepatocellular carcinoma. Hepatology 2018;67:358-380.

9. Kudo M, Izumi N, Kokudo N, Matsui O, Sakamoto M, Nakashima 0 , et al. Management of hepatocellular carcinoma in Japan: Consensus-Based Clinical Practice Guidelines proposed by the Japan Society of Hepatology (JSH) 2010 updated version. Dig Dis 2011;29:339-364.

10. Lee JM, Park JW, Choi BI. 2014 KLCSG-NCC Korea Practice Guidelines for the management of hepatocellular carcinoma: HCC diagnostic algorithm. Dig Dis 2014;32:764-777.

11. Zhang BH, Yang BH, Tang ZY. Randomized controlled trial of screening for hepatocellular carcinoma. J Cancer Res Clin Oncol 2004; 130:417-422.

12. Chalasani N, Younossi Z, Lavine JE, Diehl AM, Brunt EM, Cusi K, et al. The diagnosis and management of non-alcoholic fatty liver disease: practice guideline by the American Association for the Study of Liver Diseases, American College of Gastroenterology, and the American Gastroenterological Association. Am J Gastroenterol 2012;107:811-826.

13. Sangiovanni A, Prati GM, Fasani P, Ronchi G, Romeo R, Manini M, et al. The natural history of compensated cirrhosis due to hepatitis C virus: a 17-year cohort study of 214 patients. Hepatology 2006:43:1303-1310.

14. Ioannou GN, Splan MF, Weiss NS, McDonald GB, Beretta L, Lee SP. Incidence and predictors of hepatocellular carcinoma in patients with cirrhosis. Clin Gastroenterol Hepatol 2007;5:938-945.e4.

15. Bruno S, Stroffolini T, Colombo M, Bollani S, Benvegnu L, Mazzella $G$, et al. Sustained virological response to interferon-alpha is 
associated with improved outcome in HCV-related cirrhosis: a retrospective study. Hepatology 2007;45:579-587.

16. Sung JJ, Tsoi KK, Wong VW, Li KC, Chan HL. Meta-analysis: treatment of hepatitis $B$ infection reduces risk of hepatocellular carcinoma. Aliment Pharmacol Ther 2008;28:1067-1077.

17. European Association For the Study of the Liver. EASL clinical practice guidelines: management of chronic hepatitis $B$ virus infection. J Hepatol 2012;57:167-185.

18. Yoshida H, Shiratori Y, Moriyama M, Arakawa Y, Ide T, Sata M, et al. Interferon therapy reduces the risk for hepatocellular carcinoma: national surveillance program of cirrhotic and noncirrhotic patients with chronic hepatitis C in Japan. IHIT Study Group. Inhibition of Hepatocarcinogenesis by Interferon Therapy. Ann Intern Med 1999;131:174-181.

19. Lok AS, Seeff LB, Morgan TR, di Bisceglie AM, Sterling RK, Curto $\mathrm{TM}$, et al. Incidence of hepatocellular carcinoma and associated risk factors in hepatitis C-related advanced liver disease. Gastroenterology 2009;136:138-148.

20. Fattovich G, Stroffolini T, Zagni I, Donato F. Hepatocellular carcinoma in cirrhosis: incidence and risk factors. Gastroenterology 2004;127(5 Suppl 1):S35-50.

21. European Association for the Study of the Liver (EASL); European Association for the Study of Diabetes (EASD); European Association for the Study of Obesity (EASO). EASL-EASD-EASO Clinical Practice Guidelines for the management of non-alcoholic fatty liver disease. Obes Facts 2016:9:65-90.

22. Singal $A G$, Pillai $A$, Tiro J. Early detection, curative treatment, and survival rates for hepatocellular carcinoma surveillance in patients with cirrhosis: a meta-analysis. PLoS Med 2014;11:e1001624.

23. Costentin CE, Layese R, Bourcier V, Cagnot C, Marcellin P, Guyader $D$, et al. Compliance with hepatocellular carcinoma surveillance guidelines associated with increased lead-time adjusted survival of patients with compensated viral cirrhosis: a multi-center cohort study. Gastroenterology 2018;155:431-442.e10.

24. Moon AM, Weiss NS, Beste LA, Su F, Ho SB, Jin GY, et al. No association between screening for hepatocellular carcinoma and reduced cancer-related mortality in patients with cirrhosis. Gastroenterology 2018;155:1128-1139.e6.

25. Cadier B, Bulsei J, Nahon P, Seror O, Laurent A, Rosa I, et al. Early detection and curative treatment of hepatocellular carcinoma: a cost-effectiveness analysis in France and in the United States. Hepatology 2017;65:1237-1248.

26. Goutte N, Sogni P, Bendersky N, Barbare JC, Falissard B, Farges 0. Geographical variations in incidence, management and survival of hepatocellular carcinoma in a Western country. J Hepatol 2017;66:537-544.

27. Sarasin FP, Giostra E, Hadengue A. Cost-effectiveness of screening for detection of small hepatocellular carcinoma in western patients with Child-Pugh class A cirrhosis. Am J Med 1996;101:422-434.
28. Cucchetti A, Trevisani F, Cescon M, Ercolani G, Farinati F, Poggio $P D$, et al. Cost-effectiveness of semi-annual surveillance for hepatocellular carcinoma in cirrhotic patients of the Italian liver cancer population. J Hepatol 2012;56:1089-1096.

29. Trevisani F, Santi V, Gramenzi A, Di Nolfo MA, Del Poggio P, Benvegnu $L$, et al. Surveillance for early diagnosis of hepatocellular carcinoma: is it effective in intermediate/advanced cirrhosis? Am J Gastroenterol 2007;102:2448-2457.

30. Ruggeri M. Hepatocellular carcinoma: cost-effectiveness of screening: a systematic review. Risk Manag Healthc Policy 2012;5:49-54.

31. Bolondi L, Sofia S, Siringo S, Gaiani S, Casali A, Zironi G, et al. Surveillance programme of cirrhotic patients for early diagnosis and treatment of hepatocellular carcinoma: a cost effectiveness analysis. Gut 2001;48:251-259.

32. Patel D, Terrault NA, Yao FY, Bass NM, Ladabaum U. Costeffectiveness of hepatocellular carcinoma surveillance in patients with hepatitis C virus-related cirrhosis. Clin Gastroenterol Hepatol 2005:3:75-84.

33. Kim CK, Lim JH, Lee WJ. Detection of hepatocellular carcinomas and dysplastic nodules in cirrhotic liver: accuracy of ultrasonography in transplant patients. J Ultrasound Med 2001;20:99-104.

34. Singal $A$, Volk ML, Waljee $A$, Salgia $R$, Higgins $P$, Rogers $M A$, et al. Meta-analysis: surveillance with ultrasound for early-stage hepatocellular carcinoma in patients with cirrhosis. Aliment Pharmacol Ther 2009;30:37-47.

35. Tzartzeva K, Obi J, Rich NE, Parikh ND, Marrero JA, Yopp A, et al. Surveillance imaging and alpha fetoprotein for early detection of hepatocellular carcinoma in patients with cirrhosis: a meta-analysis Gastroenterology 2018;154:1706-1718.e1.

36. Sato T, Tateishi R, Yoshida H, Ohki T, Masuzaki R, Imamura J, et al. Ultrasound surveillance for early detection of hepatocellular carcinoma among patients with chronic hepatitis C. Hepatol Int 2009:3:544-550

37. Pocha C, Dieperink E, McMaken KA, Knott A, Thuras P, Ho SB. Surveillance for hepatocellular cancer with ultrasonography vs. computed tomography: a randomised study. Aliment Pharmacol Ther 2013;38:303-312.

38. Lee YJ, Lee JM, Lee JS, Lee HY, Park BH, Kim YH, et al. Hepatocellular carcinoma: diagnostic performance of multidetector CT and MR imaging-a systematic review and meta-analysis. Radiology 2015;275:97-109.

39. Besa C, Lewis S, Pandharipande PV, Chhatwal J, Kamath A, Cooper $N$, et al. Hepatocellular carcinoma detection: diagnostic performance of a simulated abbreviated MRI protocol combining diffusion-weighted and T1-weighted imaging at the delayed phase post gadoxetic acid. Abdom Radiol (NY) 2017;42:179-190.

40. Marks RM, Ryan A, Heba ER, Tang A, Wolfson TJ, Gamst AC, et al. Diagnostic per-patient accuracy of an abbreviated hepatobiliary 
phase gadoxetic acid-enhanced MRI for hepatocellular carcinoma surveillance. AJR Am J Roentgenol 2015;204:527-535.

41. Kim YK, Kim YK, Park HJ, Park MJ, Lee WJ, Choi D. Noncontrast MRI with diffusion-weighted imaging as the sole imaging modality for detecting liver malignancy in patients with high risk for hepatocellular carcinoma. Magn Reson Imaging 2014;32:610-618.

42. Marrero JA, Feng Z, Wang Y, Nguyen MH, Befeler AS, Roberts LR, et al. Alpha-fetoprotein, des-gamma carboxyprothrombin, and lectin-bound alpha-fetoprotein in early hepatocellular carcinoma. Gastroenterology 2009;137:110-118.

43. Marrero JA, Su GL, Wei W, Emick D, Conjeevaram HS, Fontana RJ, et al. Des-gamma carboxyprothrombin can differentiate hepatocellular carcinoma from nonmalignant chronic liver disease in american patients. Hepatology 2003;37:1114-1121.

44. Sherman M. Serological surveillance for hepatocellular carcinoma: time to quit. J Hepatol 2010;52:614-615.

45. Andersson KL, Salomon JA, Goldie SJ, Chung RT. Cost effectiveness of alternative surveillance strategies for hepatocellular carcinoma in patients with cirrhosis. Clin Gastroenterol Hepatol 2008;6:14181424.

46. Trinchet JC, Chaffaut C, Bourcier V, Degos F, Henrion J, Fontaine H, et al. Ultrasonographic surveillance of hepatocellular carcinoma in cirrhosis: a randomized trial comparing 3-and 6-month periodicities. Hepatology 2011;54:1987-1997.

47. Sangiovanni A, Del Ninno E, Fasani P, De Fazio C, Ronchi G, Romeo R, et al. Increased survival of cirrhotic patients with a hepatocellular carcinoma detected during surveillance. Gastroenterology 2004;126:1005-1014.

48. Santagostino E, Colombo M, Rivi M, Rumi MG, Rocino A, Linari S, et al. A 6-month versus a 12-month surveillance for hepatocellular carcinoma in 559 hemophiliacs infected with the hepatitis $C$ virus. Blood 2003; 102:78-82.

49. Santi V, Trevisani F, Gramenzi A, Grignaschi A, Mirici-Cappa F, Del Poggio $P$, et al. Semiannual surveillance is superior to annual surveillance for the detection of early hepatocellular carcinoma and patient survival. J Hepatol 2010;53:291-297.

50. Davila JA, Weston A, Smalley W, El-Serag HB. Utilization of screening for hepatocellular carcinoma in the United States. J Clin Gastroenterol 2007;41:777-782.

51. Davila JA, Henderson L, Kramer JR, Kanwal F, Richardson PA, Duan Z, et al. Utilization of surveillance for hepatocellular carcinoma among hepatitis C virus-infected veterans in the United States. Ann Intern Med 2011;154:85-93.

52. Giannini EG, Cucchetti A, Erroi V, Garuti F, Odaldi F, Trevisani F. Surveillance for early diagnosis of hepatocellular carcinoma: how best to do it? World J Gastroenterol 2013;19:8808-8821.

53. Goldberg DS, Taddei TH, Serper M, Mehta R, Dieperink E, Aytaman A, et al. Identifying barriers to hepatocellular carcinoma surveillance in a national sample of patients with cirrhosis. Hepatology 2017;65:864-874.

54. Farvardin S, Patel J, Khambaty M, Yerokun OA, Mok H, Tiro JA, et al. Patient-reported barriers are associated with lower hepatocellular carcinoma surveillance rates in patients with cirrhosis. Hepatology 2017;65:875-884.

55. Atiq 0, Tiro J, Yopp AC, Muffler A, Marrero JA, Parikh ND, et al. An assessment of benefits and harms of hepatocellular carcinoma surveillance in patients with cirrhosis. Hepatology 2017;65:11961205.

56. Singal AG, Tiro JA, Marrero JA, McCallister K, Mejias C, Adamson $B$, et al. Mailed outreach program increases ultrasound screening of patients with cirrhosis for hepatocellular carcinoma. Gastroenterology 2017;152:608-615.e4.

57. Kudo M. Japan's successful model of nationwide hepatocellular carcinoma surveillance highlighting the urgent need for global surveillance. Liver Cancer 2012;1:141-143.

58. Yoon SK, Chun HG. Status of hepatocellular carcinoma in South Korea. Chin Clin Oncol 2013;2:39. 